\title{
ALTAR FUNERARIO CON PVLVINI DE LA CIUDAD DE CLUNIA
}

\section{FUNERARY ALTAR WITH PVLVINI IN THE CITY OF CLUNIA}

\author{
M. a Ángeles Gutiérrez Behemerid \\ Universidad de Valladolid \\ angeles@fyl.uva.es
}

DOI: $10.1387 /$ veleia.14991

\begin{abstract}
Resumen: Se estudia un fragmento de pulvino que correspondería al único altar funerario de estas características hallado en el convento cluniense. Sus rasgos tipológicos y formales permiten su vinculación con pulvini del nordeste peninsular y, en especial, con varios de Barcelona y Tarragona.

Palabras clave: Pulvinus, altar funerario, época romana, Clunia.

Abstract: A study is carried out of a fragment of pulvino corresponding to the only funerary altar of this kind to be found in the convent at Clunia. Its typological and formal features link it to the pulvini found in the north east of the peninsula and, particularly, to several in Barcelona and Tarragona.

Keywords: pulvinus, funerary altar, Roman period, Clunia.
\end{abstract}

Recibido: 25-05-2015

Informado: 05-06-2015

Definitivo: 30-06-2015

\section{INTRODUCCIÓN}

Entre los materiales procedentes de la ciudad de Clunia se encuentra un fragmento arquitectónico recuperado con ocasión de la demolición de una casa en Peñalba de Castro y que, en nuestra opinión, pudo formar parte de un monumento funerario en forma de altar coronado por pulvini (Gutiérrez 2003, 153).

Se trata, en concreto, del frente y dorsal de un pulvino cilíndrico con alargamiento lateral que corresponde al lado derecho de un altar (Figura 1a y b). El círculo del pulvino, delimitado con un pequeño reborde en saliente, está enteramente ocupado por un gran florón formado por una doble corola de pétalos en torno a un botón central; la inferior está formada por ocho pequeñas hojitas alargadas con nervadura central, y la superior con ocho largos lóbulos de acanto, articulados en pequeñas digitaciones lanceoladas, con una profunda incisión central a modo de nervadura. El frente del alargamiento lateral del pulvino, apoyado en su parte inferior en un listel plano, se decora con tres filas de hojitas de laurel imbricadas. El lateral, conservado solo en parte, ofrece una ornamentación de varias hileras de hojitas imbricadas, prácticamente idénticas a las del frente, lanceoladas, con terminación redondeada y la parte central de la hoja fuertemente resaltada. Es posible que, como sucede habitualmente, se unieran en el centro mediante un balteo (Figura 2a y b). La pieza está fabricada en caliza local. 

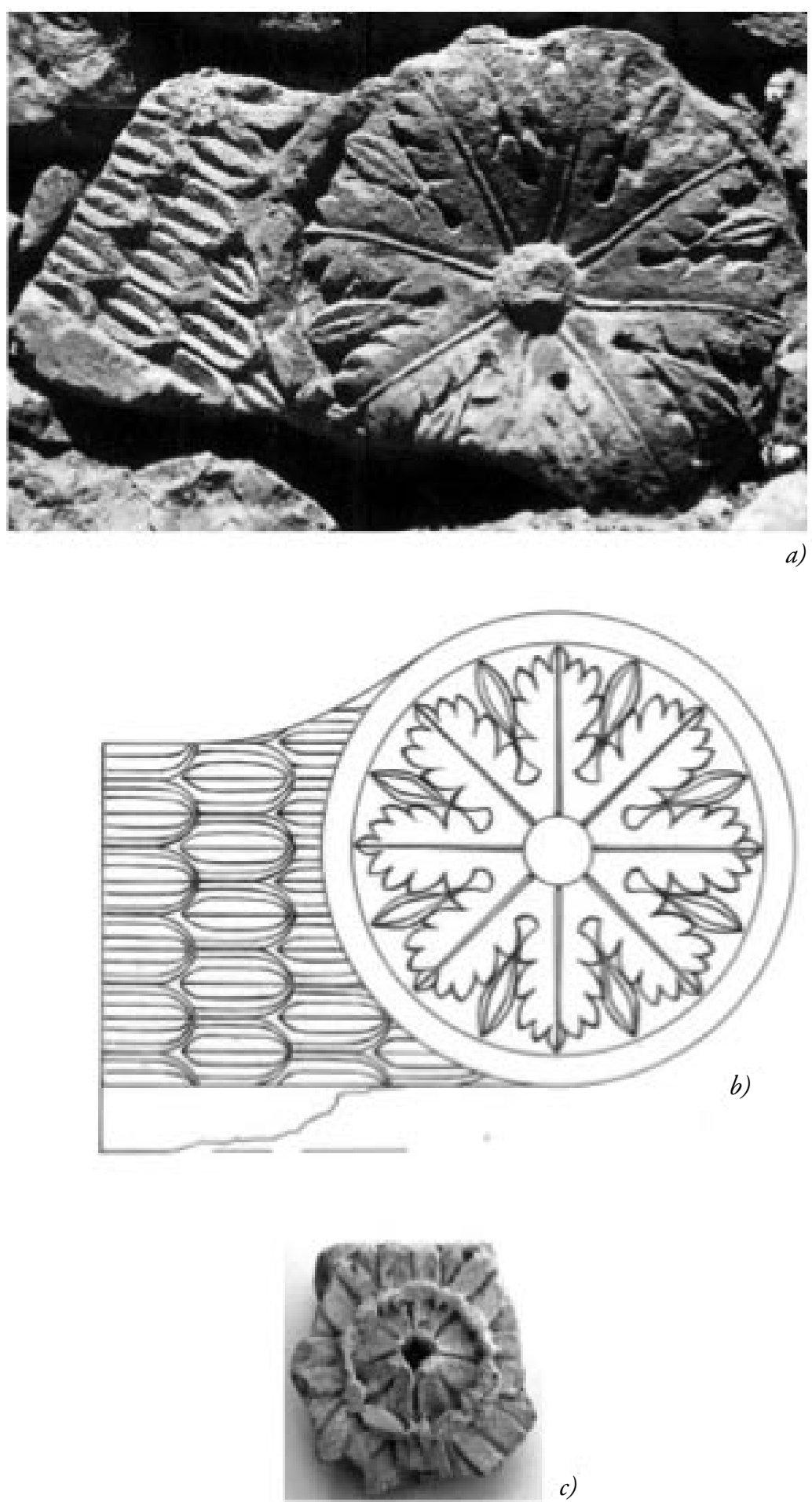

Figura I. 1a) Pulvino. Fotografía P. de Palol 1958. 1b) Frente del pulvino. Dibujo A. Rodríguez. 1c) Posible metopa decorada con un florón 

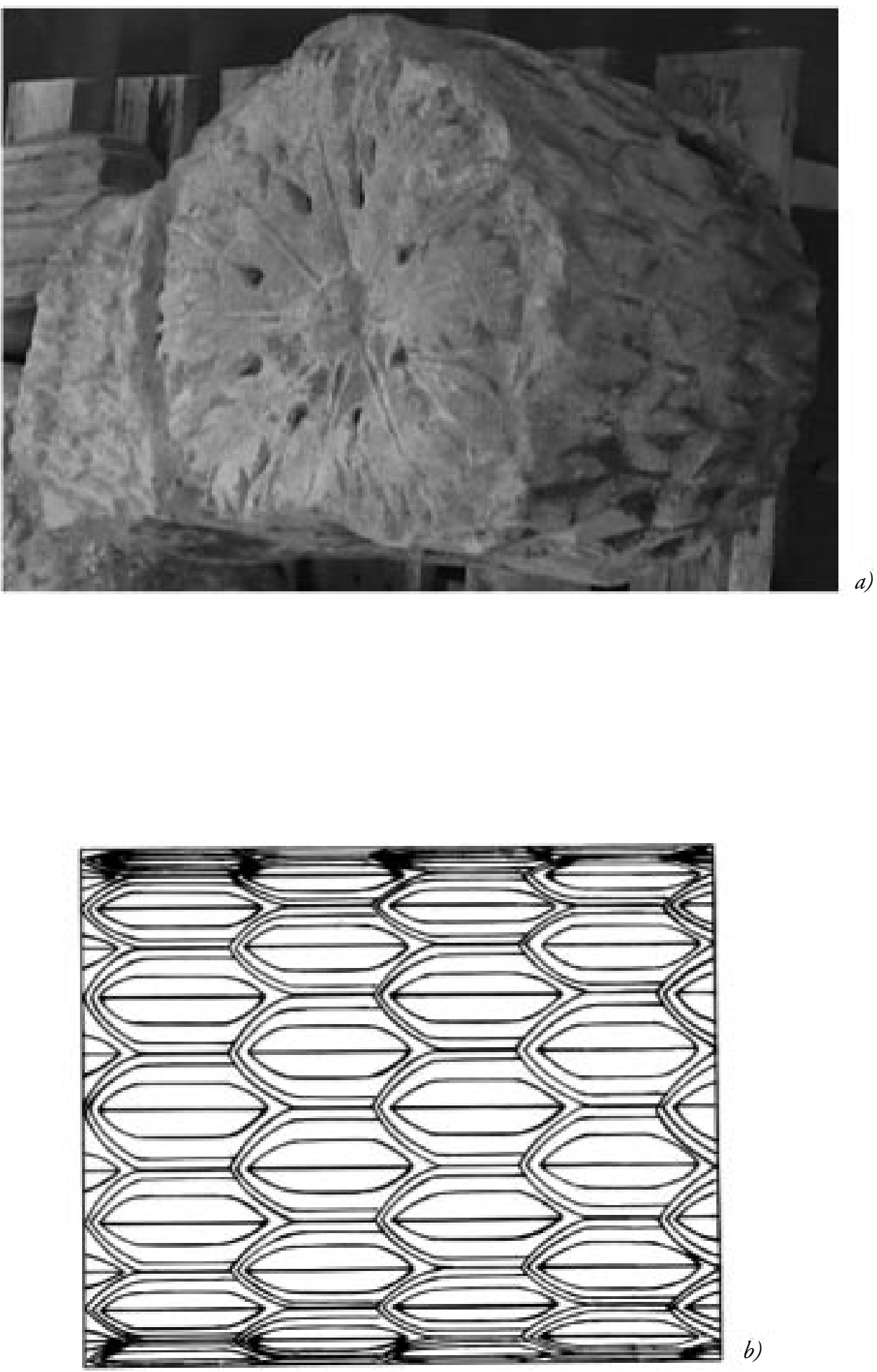

Figura 2. 2a) Frente y dorsal del pulvino. 2b) Dorsal pulvino. Dibujo A. Rodríguez 


\section{Sobre los ALtARES FunERARios}

El altar funerario ${ }^{1}$ contó con una importante presencia tanto en el mundo itálico como en las provincias occidentales del imperio. Su origen se sitúa en Sicilia y en la Magna Grecia, entre los siglos IV y III a.C., a partir de los sarcófagos monumentales de Agrigento, Siracusa o Capua. En Roma, a finales del siglo III a.C. se encontrará en el Sepulcro de los Escipiones una interpretación de este modelo, con un friso dórico y dos volutas rematando su parte superior. Su importancia tanto en Italia como en las provincias occidentales radica, sobre todo, en cierto carácter religioso que emana de este tipo de construcción. Su difusión es rápida en las colonias romanas y, si bien en un primer momento parece ser privativo de las clases medias, a partir de la mitad del siglo i a.C. se convierte en un signo distintivo de colonos y magistrados. Algo más tarde, y vinculado con la colonización militar, llega a las provincias occidentales donde se conservan un número importante de ejemplares decorados con frisos dóricos (Gros 2002, 392-395). En la península Ibérica se convertirá en una de las fórmulas más frecuentemente utilizada en la arquitectura funeraria, con una trayectoria que se inicia a finales de la época tardo-republicana y que se irá imponiendo a comienzos de la imperial, considerándose al mismo tiempo como un factor importante de romanización. Su presencia se constata tanto en el mundo urbano como en el rural.

$\mathrm{Su}$ estructura arquitectónica es muy sencilla, cuadrangular, realizada en opus quadratum. Podían estar rematados en un focus liso o bien aparecer flanqueados por los pulvini. A partir de este esquema general se contemplan dos fórmulas diferentes en esta modalidad de altar. En la más antigua, el cuerpo cuadrado o ligeramente rectangular, rematado por los pulvinos — cilíndricos o con alargamientos laterales_-, formaría la propia cámara funeraria, con acceso al interior. En la segunda variante, que incluye los altares con pulvinos de menor tamaño, el cuerpo macizo sería el coronamiento de la cámara sepulcral. El primero de los tipos mencionados se desarrollaría en Italia a finales del siglo ir a.C. mientras que el segundo correspondería a un momento posterior. Con respecto a la decoración del cuerpo del altar, existen otras posibilidades ya sean pilastras en los ángulos coronadas por distintas variantes de capitel o frisos dóricos. Entre las pilastras podía haber otros motivos ornamentales como guirnaldas u otros elementos que hicieran referencia a los cargos públicos desempeńados en vida por el difunto (Beltrán 2004,130; Osuna 2010, 106-109).

Para el análisis de la pieza cluniense se ha tomado como referencia el grupo del nordeste peninsular y, de modo especial, el que incluye los altares de Barcino y Tarraco ya que es con estos dos ámbitos con los que nuestra pieza muestra una mayor proximidad estilística y tipológica. Hay que seńalar también que ese conjunto fue el primero en ser conocido y estudiado y, que por lo tanto, es el que ha marcado las pautas para el análisis de los pulvinos de la Península, sirviendo aún de referencia los estudios llevados a cabo por Balil, aunque en algunos aspectos — su cronología, por ejemplo- hayan sido si no superados sí matizados posteriormente.

En relación a la forma que pueden adoptar los pulvinos y, siguiendo las directrices señaladas por Balil en su estudio sobre los Gorgoneia de Barcino (Balil 1979, 63-70), se han diferenciado dos tipos de frentes. Los ejemplos más tempranos y menos frecuentes muestran el frente del pulvino circular y es posible que pudieran relacionarse con los frisos dóricos; sus paralelos se encontrarían en altares itálicos como, por ejemplo, los de la Via Appia en Roma. La segunda variante incluye aquellos pulvinos que presentan un alargamiento hacia la parte central del monumento, siendo los alta-

1 Estudios detallados entre otros en: Beltrán Fortes 1990, 2004; Clavería 2008; Gros 2001, 2; Hesberg 1994. 
res de Neumagen los ejemplos más ilustrativos de este tipo. Esta modalidad se convertirá en la más representativa de los altares funerarios del nordeste peninsular pero al mismo tiempo gozará de una amplia difusión en el resto de la península Ibérica, a excepción de la zona levantina. Para Balil ambas fórmulas serían dependientes de las corrientes artísticas que se difunden desde la península Itálica hasta el Valle del Po y la Galia Narbonense, poniendo así de manifiesto su conexión con las producciones itálicas y galas. Fija su datación dentro de un amplio abanico cronológico que abarca desde fines del siglo i a.C. hasta el s. III d.C., a tenor de las semejanzas entre los pulvinos del segundo grupo y los de Neumagen (Balil 1979, 63; Clavería 2008, 349-351).

La decoración de los pulvinos hispanos responde básicamente a tres modelos en virtud de las variaciones ornamentales que se desarrollan en los frentes. El grupo más numeroso consiste en una gran cabeza de Gorgona ocupando todo el círculo del pulvino. Esta modalidad se puede considerar casi exclusiva de Barcino, con diferencias estilísticas e iconográficas entre las Gorgonas, si bien cuenta también con algunos ejemplos en Tarraco y en el sur peninsular. La segunda variante muestra un elemento vegetal - rosetas o florones- en lugar del gorgoneion. A estas dos representaciones mayoritarias se añaden las cabezas-retrato de algunas piezas, por ejemplo, de la Bética. La decoración de las partes dorsales de los pulvinos es más homogénea en todas las áreas peninsulares. Consiste, en líneas generales, en varias hileras de hojitas imbricadas de laurel que se unen en el centro mediante un balteo con distintos motivos, siendo el más frecuente el nudo de Hércules. En ocasiones se pueden presentar simplemente desbastados, sin ninguna ornamentación. Se han señalado igualmente diferencias con respecto a la talla y a la ejecución de los laterales pulvinares. La ornamentación de los pulvini ha permitido establecer una clara distinción entre los de Barcelona y los de Tarragona en función de una mayor dependencia de los modelos itálicos, metropolitanos, en el caso de Tarragona, mientras que los de Barcino acusan un mayor provincialismo (Claveria 2008, 361-362).

Estos monumentos funerarios no se pueden considerar exclusivos de una determinada zona geográfica ya que su área de expansión se extiende prácticamente a toda la cuenca mediterránea, con una amplia documentación en la Galia, especialmente en la Narbonense. Allí ofrecen como rasgo distintivo la presencia en el centro del pulvino de una cabeza-retrato en lugar de la Gorgona. En Germania, donde su presencia parece ser algo más tardía, el rasgo más definitorio es mostrar un frontón triangular entre los pulvini. Aún se pueden añadir ejemplos aislados en Britannia y en el Norte de Africa (Clavería 2008, 351-352). Su presencia y difusión se ha venido relacionando con la colonización militar del s. I a.C., ya que su incidencia es mayor precisamente en aquellos lugares donde más intensa fue esta. Su expansión se explica, también, en relación al ambiente cultural en el que esta colonización se llevó a cabo, valorando las influencias tardo-helenísticas existentes en aquellas zonas en las que en el siglo ir a.C. se había extendido la cultura helenístico-itálica. El caso hispano entraría, por tanto, de lleno dentro de esta misma consideración (Torelli 1968, 47-48; Joulia 1988, 215-216; Gutiérrez 1990, 212-213).

Además de los pulvini estos altares pudieron presentar, como ya se ha mencionado, otros elementos decorativos, entre los que cabe destacar los frisos dóricos con triglifos y metopas ocupadas con diferentes motivos, tanto de carácter vegetal como cultual. Tradicionalmente se ha planteado la relación de los frisos dóricos con esta modalidad de altar funerario y, de hecho, son numerosos los ejemplos de frisos dóricos que aparecen asociados a estas construcciones señalando, a la vez, su amplia difusión.

Es difícil, sin embargo, asociar, en líneas generales, los frisos dóricos a los altares con pulvinos. En el caso catalán, por ejemplo, faltan datos acerca del contexto en el que fueron encontrados que permitieran una atribución de forma más fiable al ámbito funerario; a ello se añade que son pocos 
los frisos dóricos conocidos en relación al número mucho mayor de pulvinos. No se puede olvidar, tampoco, que los frisos dóricos pueden decorar otro tipo de monumentos como los turriformes. La riqueza decorativa de las piezas localizadas en Cataluña permite pensar en la presencia tanto de frisos dóricos como, más probablemente, de frisos de roleos. Otras ciudades peninsulares, caso de Segobriga o de Mérida, que cuentan también con un número importante de pulvini, no han proporcionado sin embargo frisos dóricos, al contrario de lo que sucede por ejemplo en la Bética, donde los frisos dóricos acompańan a otros elementos arquitectónicos — pilastras angulares con guirnaldas- o bien han sido sustituidos por otro tipo de ornamentación como guirnaldas de acanto o metopas (Beltrán 2004, 132).

Con respecto al coronamiento superior de estos altares, la propia disposición de los pulvinos deja un espacio vacío intermedio que pudo haber sido ocupado por un pequeño frontón triangular, tal y como aparece en varios altares de Neumagen, que muestran un frontón decorado con una máscara que en ningún caso sobrepasa la altura de los pulvinos. Hay que señalar, sin embargo, que no hay ninguna base para pensar que los pulvinos hispanos se remataran con un frontón tal y como se restituyó en algunos altares barceloneses (Gamer 1989, 137a). De hecho, no se ha encontrado ningún elemento de estas características asociado a pulvinos que confirme dicha relación (Beltrán 2004, 133-34). Diferente es el caso de algunos alteres jienenses coronados por un frontón monolítico (Beltrán 1990, 19-22). Otra propuesta es la que se contempla para los pulvinos emeritenses y que consiste en no dejar ningún espacio intermedio entre el alargamiento de los pulvinos, considerando varias opciones al respecto (Nogales, Márquez 2002, 126, 3, a-c).

La cronología de los pulvinos hispanos ha sido objeto de debate, ya que no es fácil de precisar en la medida que se desconoce el contexto de aparición de gran parte de ellos. Beltrán lleva su inicio a la época augustea, con un momento álgido durante la julio-claudia, manteniéndose todo el siglo i y con una presencia aún en el siglo in d.C. Un apoyo para esta temprana cronología es el hecho de que se haya utilizado la piedra local para su realización (Hesberg 1994, 166; Gros 2002, 395; Beltrán 2004, 128). De hecho, salvo poquísimas excepciones —algún ejemplar de Tarragona o de la Betica-, el empleo del mármol es muy escaso.

El altar funerario cuenta, pues, con una presencia importante en la Península. Si en los primeros momentos de la investigación su área de expansión quedaba restringida al NE peninsular, especialmente a Barcelona y, en menor medida, a Tarragona, este ámbito inicial se ha visto ampliado con otros ejemplos en el valle del Ebro, desde Zaragoza y Teruel hasta Navarra y la Rioja, y especialmente en el sur de la Península, donde se localiza uno de los focos más importantes. Se conocen también otros conjuntos aislados en la Lusitania y un grupo importante en el interior de la Meseta sur, concentrados en la ciudad de Segobriga. La zona levantina cuenta con algunas piezas más tardías, fechadas a partir de la segunda mitad del s. I d.C. y, finalmente, en el SE peninsular, en torno a Albacete, se localiza un importante núcleo de cronología temprana (Beltrán 2004, 102). A estos ámbitos geográficos se podría añadir la Meseta, con el ejemplo de la ciudad de Clunia. El área de dispersión de este tipo de altar con pulvinos viene a coincidir con la de aquellos territorios en los que se documentan también otros tipos de construcciones funerarias de carácter monumental y en tal sentido hemos de señalar que el convento cluniense cuenta con un importante repertorio de ellos.

Se han formulado diferentes planteamientos con respecto a la difusión de esta construcción. Se ha propuesto una doble órbita de influencias que incluiría, por un lado, a la Tarraconense y al valle del Ebro, en la que priman los influjos galos, mientras que en la Bética se acusaría más la influencia norteafricana. Esta hipótesis, sin embargo, hoy día no se mantiene y se prefiere explicar su presencia en función del proceso de romanización de la península Ibérica y de los cambios sociales que se producen a lo largo del siglo i d.C. (Beltrán 2004, 136). 


\section{El PUlvino Cluniense}

El ejemplo cluniense se puede incluir en el segundo de los tipos mencionado por Balil (Balil 1979, 63) que es el más documentado y representativo de los altares funerarios de la Península y, especialmente, del nordeste peninsular, ámbito que, como ya seńalamos, nos interesa de modo especial. Esta pieza puede relacionarse tanto desde el punto de vista estilístico como tipológico con algunos procedentes de Barcelona y, especialmente, de Tarragona con los que comparte tanto la ornamentación del centro del pulvino con un gran florón como también la del dorsal (Clavería 2008,11-14; Garrido 2011, 373,374, 379 entre otros). Hay que añadir, además, una particularidad que afecta a algunos pulvinos de Tarraco y que se manifiesta igualmente en este ejemplo y es el que la longitud del frente del pulvino sea más corta con respecto a los de Barcelona (Clavería 2008, $381,12,31)$.

$\mathrm{Al}$ margen de las analogías tipológicas mencionadas, son escasísimos, por no decir casi nulos, los paralelos que se pueden aducir para el florón que decora el centro del pulvino, ya que apenas hay ejemplos que muestren unos rasgos similares al cluniense y, menos aún, en esta posición; en realidad, su presencia es mayor en la ornamentación de frisos dóricos, casetones de cornisa o, incluso, en frisos de roleos de acanto. Dentro del ámbito hispano el modelo más próximo es un friso dórico, de carácter funerario, procedente de "La Chica», en Mengíbar (Jaén) (Weiss 2000, 30 a, 40), así como un florón de cornisa de las termas de Munigua aunque con una datación bastante posterior (Ahrens 2004, 391, 9, 28c).

Otros ejemplos hispanos, con los que se podría establecer una cierta relación, son los florones que aparecen tanto en algún friso dórico como en frisos funerarios de roleos de Barcelona; hay que señalar, sin embargo, que existen diferencias en cuanto a la configuración del tipo de acanto, más cercano al estilo del segundo triunvirato en el caso barcelonés, mientras que la pieza cluniense acusa un mayor naturalismo (Gutiérrez 1999, I,1; Garrido 2011, 1-2).

Fuera de la Península es posible encontrar algunos florones afines que, en ningún caso, corresponden a la decoración pulvinar. Así, en Pola hay uno similar en un sofito correspondiente a una construcción funeraria datada en torno a la mitad del siglo I d.C. (Cavalieri 1978, 62,2). Una mayor relación estilística se aprecia con el mundo galo pero siempre como ornamentación de casetones de cornisa o de las metopas de frisos dóricos, tal y como ocurre en Saintes (Tardy 1994, 13 y 45-46), en Glanum (Gros 1981, 9, 13, 18) en Mandeure (Blin 2011, 6) o en Narbona (Janon 1986, 2); si bien en alguno de los casos citados, tal y como sucedía en los frisos barceloneses, con una configuración simétrica del acanto.

Hay que señalar que tampoco se han hallado en la propia ciudad de Clunia otros fragmentos arquitectónicos con los que poder vincular estilísticamente este pulvino ya que, como venimos señalando, se aparta de la tónica habitual de las producciones clunienses.

Su análisis, tanto estilístico como tipológico, aboga por una cronología relativamente temprana para la ciudad de Clunia y que se podría situar a comienzos del periodo julio-claudio. En los momentos iniciales de la actividad edilicia en la ciudad se aprecia, además, una mayor conexión con los modelos itálicos, especialmente a través de la Narbonense — relieves de armas por ejemploy en cierta manera también con Tarragona, tal y como pone de manifiesto algún capitel corintio. Correspondería, por tanto, a un momento previo al comienzo de la actividad del taller cluniense, que se sitúa a partir de la mitad del siglo i d.C. A favor de esta cronología julio-claudia temprana abogan también los paralelos que se han aducido - Narbona, Barcelona, Tarragona - tanto desde el punto de vista de la tipología del pulvino como con respecto al florón y a la decoración del frente y dorsal. En suma, esta pieza corrobora de nuevo una cierta influencia de los modelos de 
Tarraco en un momento en el que se observa una influencia más directa de las formas itálicas en la ciudad.

\section{Restitución}

Finalmente, queda abordar su restitución lo que no deja de ser aventurado, pues sólo disponemos de este elemento arquitectónico para llevarla a cabo y desconocemos sus dimensiones (Figura 3). Señalar además que no tenemos constancia de que exista cualquier tipo de datos en relación a esta pieza, totalmente descontextualizada. Tomando como referencia ejemplos similares, se trataría de una construcción cuadrangular, de pequeño/mediano tamaño, sobre un zócalo no muy alto y coronado por dos pulvini con alargamiento lateral hacia el centro del altar. La cámara sepulcral estaría en el propio cuerpo del altar siguiendo la fórmula habitual empleada en otras restituciones similares. No hay ninguna base que permita pensar en un pequeño frontón triangular en el espacio libre entre los pulvinos.

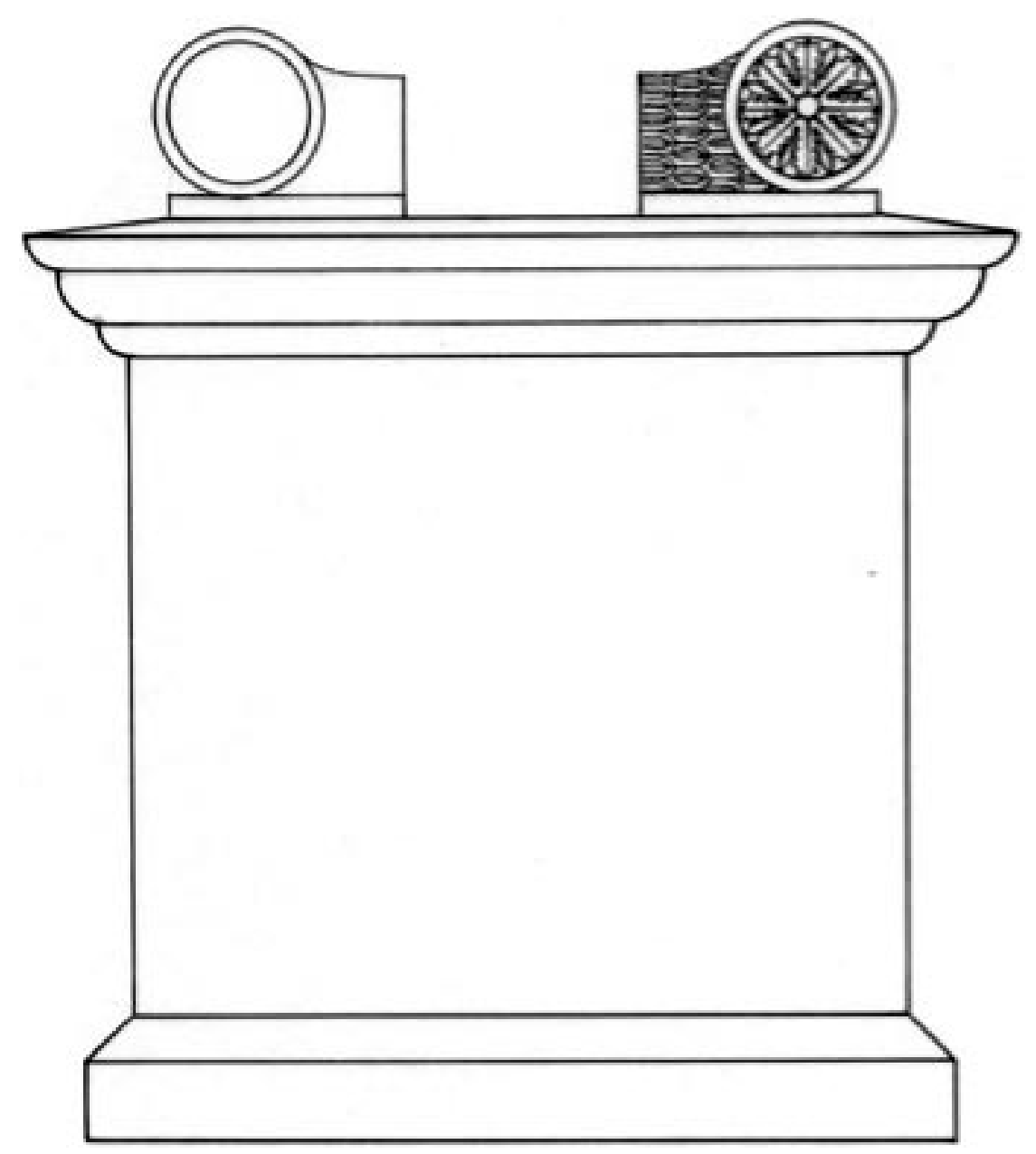

Figura 3. Restitución del altar. Dibujo A. Rodríguez 
Más difícil es hacer una propuesta de su posible tamaño ya que esta pieza debido a su fragmentación podría proporcionar únicamente datos relativos a la anchura total del monumento. Si se sigue la fórmula señalada a propósito de los altares tarraconenses con un alargamiento lateral reducido, la anchura se puede establecer en base a la longitud de los pulvinos, por cuanto estos corresponderían a un tercio de la anchura total (Clavería 2008, 381). No es posible determinar su profundidad ya que solo se conserva una parte del dorsal del pulvino.

Con respecto a su posible ornamentación caben igualmente varias hipótesis si tenemos en cuenta los motivos más habituales en este tipo de altares, que incluyen desde la presencia de un friso dórico, una guirnalda o sencillamente una superficie lisa. No podemos optar por ninguna de las posibilidades dado que en el propio yacimiento no existe o no conocemos ningún fragmento arquitectónico que se pudiera vincular con una construcción de estas características. Hay fragmentos de frisos decorados con guirnaldas de acanto pero tanto sus características estilísticas como sus dimensiones no parecen ser acordes con este tipo de altar.

A favor de la presencia de un friso dórico, y como mera hipótesis de trabajo, se podría señalar un florón encontrado en el teatro. Su estructura cuadrada y sus dimensiones lo harían apropiado para ocupar el espacio de una metopa (Figura 1c). Además, la propia tipología de la pieza, bastante particular por otro lado, no permite considerarla dentro de otras fórmulas tales como un casetón de cornisa, decoración de un sofito o florón de ábaco por ejemplo. Se trata de un florón con una doble corona de pétalos lisos alargados; la corona interna con los pétalos agrupados en dos y un motivo de perlas muy alargadas y astrágalos separando ambas coronas. En cualquier caso no hemos encontrado un paralelo si quiera aproximado para este modelo de florón.

Cabe destacar finalmente que este fragmento arquitectónico es, por el momento, el único ejemplar que puede vincularse con este tipo de construcción funeraria en el convento cluniense. En favor de su carácter funerario incide su lugar de procedencia, la actual Peñalba de Castro situada, en opinión de Palol, en la que fue una de las principales vías de acceso a la ciudad de Clunia y donde han sido encontradas un número importante de inscripciones, todas ellas de carácter funerario. De ahí que es muy posible que este lugar correspondiera a una de las necrópolis más importantes de la ciudad y, tal vez la que sirvió como lugar de enterramiento a una de las familias de la ciudad y, especialmente, a la de los Iulii (Palol, Vilella 1987, 42).

\section{BiBLIOGRAFÍA}

Ahrens, S., 2004, «Baudekor von Munigua», MM 45, 371-446.

Balil, A., 1979, «Los Gorgoneia de Barcino», Faventia 1/1, 63-70.

Beltrán Fortes, J., 1990, "Mausoleos romanos en forma de altar del Sur de la Península Ibérica», AEspA 63, 183-226.

—, 2004, "Monumenta sepulcrales en forma de altar con pulvinos de los territorios hispanorromanos: revisión de materiales y estado de la cuestión", $A E s p A 77,101-141$.

Blin, S., 2011, «Mandeure. Un programme architectural tardo-augustéen» en: Reddé, M. et al. (dir.), Aspects de la Romanisation dans l'Est de la Gaule [Col. Bibracte 21], Glux-en-Gienne, 275-286.

Cavalieri Manase, G., 1978, La decorazione architettonica romana di Aquileia, Trieste, Pola. I. L'età republicana, augustea e Giulio Claudia, Padova.

Claveria, M., 2008, «Los altares monumentales con pulvini del nordeste peninsular», Escultura Romana en Hispania V, 345-396.

Gamer, G., 1989, Formen römischer Altäre auf der Hispanischen Halbinsel, Mainz am Rhein. 
Garrido Elena, A., 2011, «Aproximación a la arquitectura funeraria de Barcino (Barcelona) en época alto imperia»» en: D. Espinosa, S. Pastor (coord.), Mors omnibus instat. Aspectos metodológicos, epigráficos y rituales de la muerte en el Occidente, Madrid, 351-372.

Gros, P., 1981, «Les Temples Géminés de Glanum. Étude preliminaire», RAN, XIV, 125-158.

—,2001, L'Architecture Romaine. 2. Maisons, palais, villas et tombeaux, Paris, 392-399.

Gutiérrez Behemerid, M. A., 1990, «Frisos dóricos funerarios en la Península Ibérica: Sistematización y cronología", BSAA, LVI, 205-213.

—,2003, La decoración arquitectónica en la Colonia Clunia Sulpicia, [Studia Archaeologica, 92], Valladolid.

Hesberg, H. von, 1994, Monumenta. I Sepolcri Romani e la loro architettura, Milano.

Janon, M., 1986, Le décor architectonique de Narbonne. Les rinceaux [Suppl. Revue Archeologique de Narbonnaise, 13], Paris.

Joulia, J. C.-L., 1988, Les frises doriques de Narbonne, [Coll. Latomus, 202], Bruxelles.

Nogales, T., Márquez, J., 2002, «Espacio y tipos funerarios en Augusta Emerita», en: D. Vaquerizo (ed.), Espacios y usos funerarios en el Occidente romano, I, Córdoba, 113-144.

Osuna, A. B., 2010, "Colonia Patricia, centro difusor de modelos: Topografía y monumentalización funerarias en Baetica» [Monografías de Arqueología Cordobesa 17], Córdoba, 116-119.

Palol, P. de, Vilella, J., 1987, Clunia II. La epigrafía de Clunia [EAE, 150], Madrid.

TARDy, D., 1994, Le décor architectonique de Saintes Antique. II. Les entablements, [Suppl. Aquitania, 7], Bordeaux.

Torelli, M., 1968, «Monumenti funerari romani con fregio dórico», DialA 2. 1, 32-54.

WeIss, Ch., 2000, «Die Steindenkmäler der Sammlung «de la Chica» in Mengíbar (Jaén) im Kontext der Sepulkralkunst des oberen Gualdalquivirtales», $M M$ 41, 253-317. 